\title{
ROBOTIC ASSISTANCE TO COUNTER IMPROVISED CBE (CHEMICAL, BIOLOGICAL, EXPLOSIVE) THREATS
}

\author{
YVAN BAUDOIN \\ Royal Military Academy \\ Belgium
}

A natural or human-caused 'disaster' usually is a vast area where massive and diverse evidences must be located, collected and transferred for analysis to a distant reference laboratory. Every single detail may be crucial for the identification of the causes and/or the criminal action of groups responsible of a potential risk caused by improvised chemical, biological and explosive devices. Nevertheless, the main difficulty is to determine which kind of evidence to look for. A detailed knowledge of similar events/contamination process could be helpful results of a threat analysis (prediction) will be useful (preparation of the plot, logistics, targeting, action, post-action steps - prevention). Cooperative multi-robot systems based on UAV and UGV equipped with modular sensors and intervention tools (detection, measurements) will carry out the screening, searching and collection of samples in the "hot zones" where the first responders could be exposed to dangerous and hazardous agents. Artificial intelligence techniques applied to the acquired data will contribute to improve detection and classification of evidentiary material and discrimination of mixed evidence, all of them critical for forensic investigations. Data fusion and artificial intelligent techniques will also be applied for predicting the dispersion patterns of some biological agents, providing in this way, a useful decision making tool for first-responders.

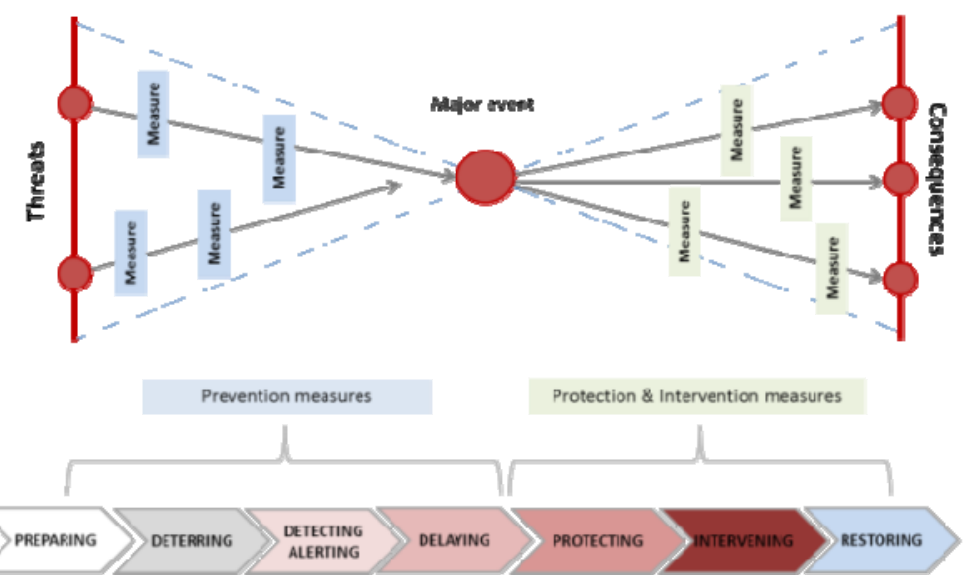

In the aftermath of a BWA of a terrorist attack, the struck area is rather difficult to access by the first responders and LEAs, and may have become hazardous, hostile or toxic, as (very partially) illustrated by the recent events in European Capitals. Establishing whether the ground can be entered safely by human beings is time consuming. A major challenge is to quickly acquire and gather in situ data and forward information about the actual situation throughout the entire system: the Robotics assistance offers practical solutions to this problem. 